\title{
Volume Phase Transition Process and Spin Diffusion in Heterogeneous Structure of Acrylamide Gels Studied by Pulsed NMR
}

\author{
Takayuki IKeHARA, Toshio Nishi, and Takafumi HaYaSHI* \\ Department of Applied Physics, School of Engineering, The University of Tokyo, \\ 7-3-1, Hongo, Bunkyo-ku, Tokyo 113, Japan \\ * Department of Computer Science and Engineering, The University of Aizu, \\ Aizuwakamatsu-shi, Fukushima 965-80, Japan
}

(Received November 2, 1995)

\begin{abstract}
Both ionized and non-ionized acrylamide gels in acetone-water mixture were studied by pulsed NMR. We investigated (i) dependence of the spin-spin relaxation time $T_{2}$ of the gels on the composition of the solvent, (ii) temporal changes of $T_{2}$ during the volume phase transition process for the ionized gels, and (iii) domain sizes of heterogeneous structures in the non-ionized gels caused by abrupt changes of the environment using the spin diffusion phenomenon. The results of the concentration dependence of $T_{2}$ showed that molecular mobility of both the solvent and the network was further depressed as the acetone content was increased in the contracted state although the volume change was small. These results show that the interaction parameter between the solvent and the network polymer has more influence on the mobility than the volume of the system. The shrinking and the swelling process of gels undergoing volume phase transition were different from each other. This reflects the behavior that the swelling and shrinking start on the boundary of the samples. The obtained domain sizes of the heterogeneous structure indicate that solid-like regions are formed on a smaller scale in the dense phase of the network. KEY WORDS Acrylamide Gel / Volume Phase Transition / Heterogeneous Structure / Pulsed Nuclear Magnetic Resonance / Spin-Spin Relaxation Time/Spin Diffusion /
\end{abstract}

Gels attract a lot of interest because of their drastic volume changes induced by the change of the environment they are placed. Ionized acrylamide gels, for example, undergo volume phase transition induced by the change in composition of the mixed solvent, temperature, $\mathrm{pH}$, and salt concentration. ${ }^{1-5}$

There have been several works on changes of molecular mobility and dynamics on volume phase transition. ${ }^{6-11}$ Katayama et al. studied ionized acrylamide gels in acetone-water mixtures by electron paramagnetic resonance. ${ }^{6}$ They observed discontinuous changes of molecular mobility at the transition point. Hu et al. studied microenvironment in acrylamide gels that undergo volume phase transition induced by $\mathrm{pH}$ and the composition of acetone-water mixtures. ${ }^{7-9}$ They showed that molecules have sufficient mobility in the collapsed state near the transition point when the transition is induced by the change of $\mathrm{pH} .{ }^{7}$ There is also a deuterium NMR study of acrylamide gels. ${ }^{10}$ A proton NMR study of $N$ isopropylacrylamide gels ${ }^{11}$ revealed that main chains of the ionized samples are mobile in the collapsed state near the transition point, while those of the nonionized ones are immobile. This was ascribed to the increased "stiffness" of the chains caused by the interaction with cations.

In these studies, molecular mobility was measured when the systems have reached the equilibrium state after the change of the environment. There have been fewer studies on dynamics during the volume phase transition process except a pulsed NMR study on the volume change process of acrylamide gels that do not undergo volume phase transition. ${ }^{12}$

A rapid change of the environment not only causes contraction of gels, but also often produces heterogeneous structures inside the gels regardless whether they undergo volume phase transition. This is because the shrinking rate, which is governed by the cooperative diffusion coefficient of the network, ${ }^{13,14}$ is too small to follow the rapid change of the environment. In such a situation, the network forms sparse and dense regions to reduce total free energy of the system. It gains contact free energy by gathering the network chains in dense regions and the obtained energy compensates the entropic loss due to the heterogeneity. Gels with large dimensions often show the above behavior since it takes a long time for them to reach the equilibrium state. ${ }^{13,14} \mathrm{Li}$ et al. treated this process as spinodal decomposition using a turbidity technique. ${ }^{15}$

Pulsed NMR is a powerful method to obtain the molecular mobility and the fractional amounts of heterogeneous phases in a sample. There are some examples applied to gels. ${ }^{16-28}$ The main quantities obtained by pulsed NMR are the spin-spin relaxation time $T_{2}$, the spin-lattice relaxation time $T_{1}$, and that in the rotating frame $T_{1 \rho}$. Since the time required for measurement is sufficiently shorter than that for the change in the system, especially for polymers, it enables us to analyze dynamics of the process. The signal of pulsed NMR for a heterogeneous system is superposition of different decay curves. One can obtain the mobility and the fractional amount of each phase by decomposing the signal. However, spin diffusion process causes energy transport between heterogeneous phases over a distance of several nanometers. This makes it ambiguous to determine the fractional amounts of heterogeneous phases in $T_{1}$ and $T_{1 \rho}$ measurement. ${ }^{19}$ Analyses of the transverse relaxation signals have been employed to decide the fractional amount for the above reason since the spin diffusion phenomenon has been considered unfavorable.

However, the spin diffusion process gives us information on the sizes of heterogeneous regions ${ }^{20-22}$ using the Goldman-Shen pulse sequence. ${ }^{23}$ This sequence 
first brings spin systems with different $T_{2}$ to different spin temperatures. Magnetization in the hard phase in a sample vanishes and that in the soft phase has a sufficient magnitude at this stage. The magnetization diffuses into the hard regions from the surrounding soft regions. After a certain diffusion time, the magnitude of magnetization in the hard phase is measured. One can obtain magnetization recovery curves in the hard phase with this procedure after several times of measurement with different diffusion time.

In this paper, we will discuss the properties of acrylamide gels in acetone-water mixture studied by pulsed NMR. After the measurement of concentration dependence of $T_{2}$, we investigated the change in molecular mobility during the volume phase transition process, both the shrinking and the swelling behavior. Domain sizes of heterogeneous phases caused by abrupt changes of the environment were also estimated with the Goldman-Shen pulse sequence. Relationship between microscopic environment, i.e., molecular mobility and macroscopic volume is discussed.

\section{EXPERIMENTAL}

We used both ionized and non-ionized acrylamide gels in this study. The ionized samples were used for studies on the volume phase transition process and the neutral ones were used for studies on heterogeneous structures created by abrupt changes of the environment, i.e., the composition of the solvent.

Ionized samples were composed with $19 \mathrm{~g}$ of the acrylamide monomer (main chain), $0.133 \mathrm{~g}$ of $N, N^{\prime}$ methylenebisacrylamide (cross-linking agent), and $1.34 \mathrm{~g}$ of sodium acrylate (ionizing substance) in water. The total volume of the solution was $100 \mathrm{ml}$. Non-ionized samples were composed with $20 \mathrm{~g}$ of the acrylamide monomer without sodium acrylate. After $N, N, N^{\prime}, N^{\prime}$ tetramethylenediamine (initiator) and ammonium peroxodisulfate (accelerator) were added, gelation took place in tubes with different inner diameters and cylindrical samples were obtained. They were $3.7 \mathrm{~mm}$ for the volume change and phase transition experiments, and $1.55 \mathrm{~cm}$ for studies on heterogeneous structures. After the completion of gelation, the samples were immersed into a large amount of water to wash away unreacted substances. Acetone-water mixtures were used as the solvent in the present experiment.

The NMR instrument was Bruker PC-20 with the resonant frequency of $20 \mathrm{MHz}$ for the proton. The signals were inputted into an $\mathrm{A} / \mathrm{D}$ converter (FIXX FSA1000SV) connected to a personal computer (NEC PC-9801VX). Spin-spin relaxation time $T_{2}$ was measured with the Carr-Purcell-Meiboom-Gill (CPMG) pulse sequence $^{24}$ in most of the experiments. The solid echo pulse sequence ${ }^{25}$ was also applied to obtain information on the short-time behavior, if needed. The values of $T_{1}$ were measured by the inversion recovery pulse sequence for samples with heterogeneous structure. The signals were analyzed by the nonlinear least-square method. The modified Goldman-Shen pulse sequence ${ }^{22}$ was employed for experiments on estimation of the sizes of heterogeneous structures. This sequence is a combination of the solid echo and the usual Goldman-Shen sequence to avoid the influence of the dead time. The schematic figure of this pulse sequence is shown in Figure 1.

\section{RESULTS}

Figure 2 shows the dependence of the swelling ratio $V / V_{0}$ (defined as the volume of the gel divided by that at gelation) of the neutral gel (a) and the ionized gel (b) on the volume percentage of acetone in the mixed solvent $c$. The volume phase transition occurred at $c=49 \%$ for the ionized sample. The composition of the solvent was changed slowly to keep transparency and homogeneity of the networks. An example of a spin-spin relaxation decay of a non-ionized gel in the solvent with $c=30 \%$ is illustrated in Figure 3. Figures 4 and 5 show the composition dependence of $T_{2}$ and the fractional amount $f$ of each phase for the neutral and the ionized samples, respectively. The NMR signals were decomposed into

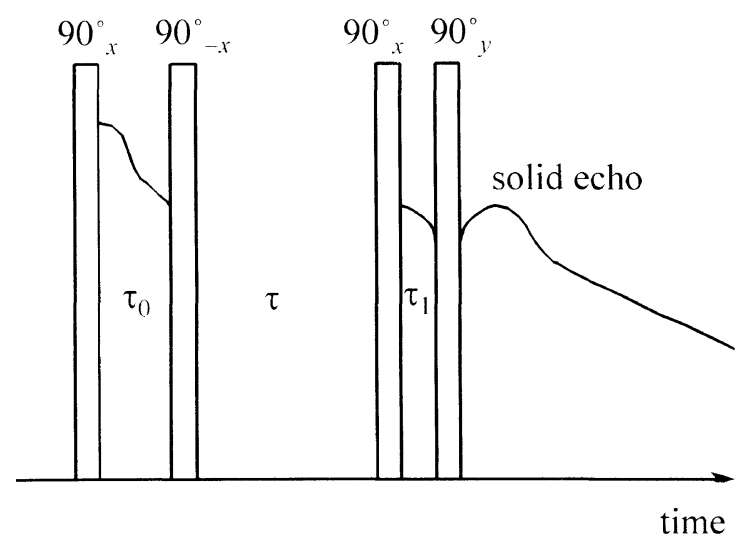

Figure 1. Schematic figure of the modified Goldman-Shen pulse sequence $\left(90_{x} \tau_{0} 90_{-x} \tau 90_{x} \tau 90_{y}\right)$. The spin diffusion takes place during the diffusion period $\tau$.
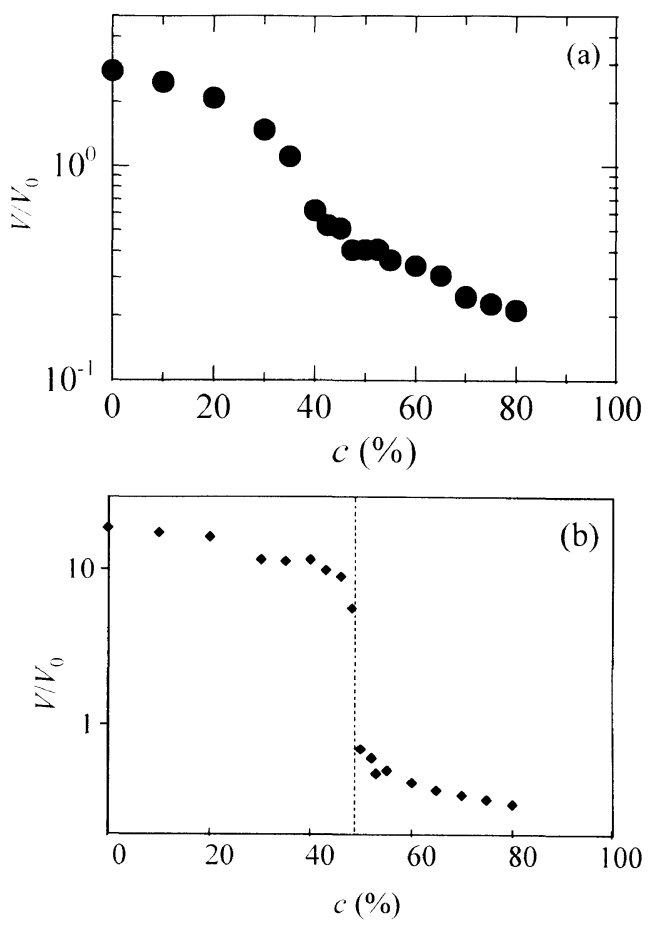

Figure 2. Dependence of the volume of the neutral gel (a) and the ionized gel (b) on acetone content of the solvent. The volume is reduced by that just after gelation. 


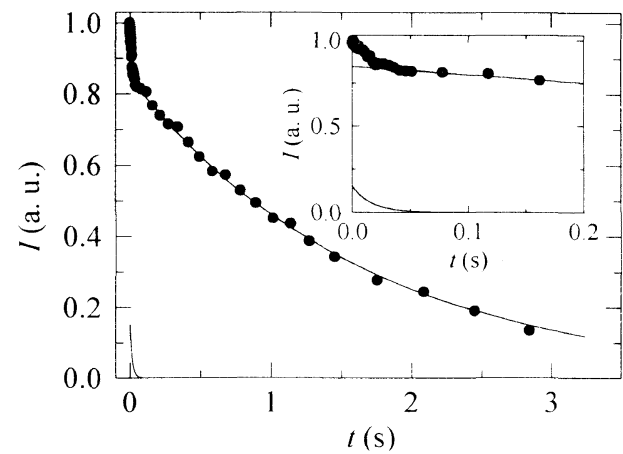

Figure 3. An example of a transverse relaxation decay curve (intensity I) decomposed into two components for a non-ionized gel in the solvent with $c=30 \%$. The dots are the data obtained by NMR and the lines are the fitted functions. The inset shows the short-time-range behavior.
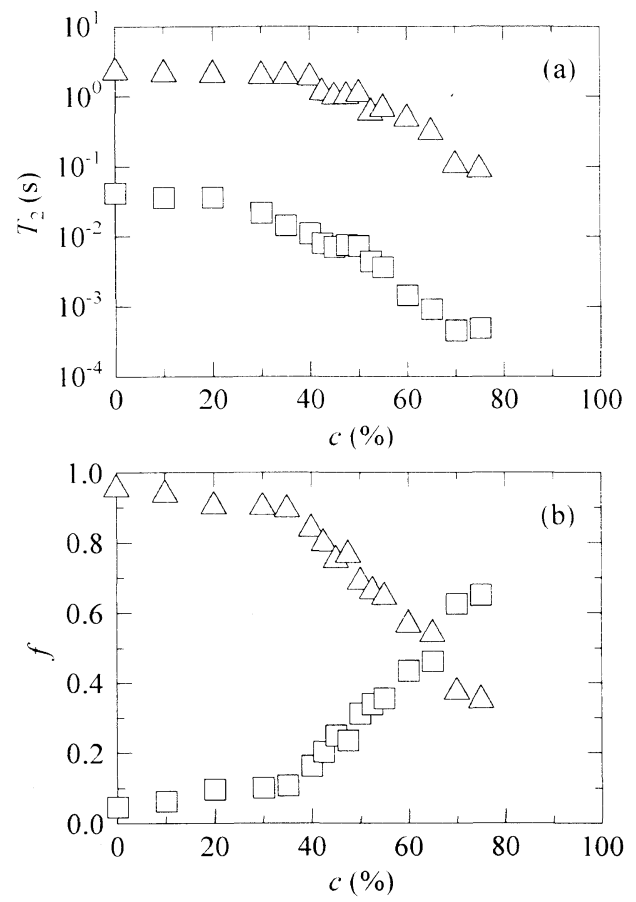

Figure 4. Dependence of $T_{2}$ (a) and fractional amount $f$ (b) of each phase in the non-ionized gel on acetone content of the mixed solvent. The NMR signals were decomposed into two components.

two components for all $c$ in both cases. Figure 6 shows the proton molar fraction of the solvent and the network calculated from the volume and mass for neutral (a) and ionized (b) gels. Since the dependence of $f$ in Figure 4(b), Figure 5(b), and Figure 6 is almost the same, the components with the long $T_{2}\left(T_{2 \mathrm{~L}}\right)$ and the short $T_{2}\left(T_{2 \mathrm{~S}}\right)$ originate from the solvent and the network polymer, respectively.

The values of $T_{2 \mathrm{~L}}$ and $T_{2 \mathrm{~S}}$ decreased a little at the volume phase transition point for the ionized sample. This is because the shrinkage suppresses molecular mobility in the collapsed state. A more interesting result is that the mobility is further suppressed with increasing $c$, especially when $c>70 \%$, although the volume change in the collapsed state is much smaller than at the transition point (Figures 2 and 5). The non-ionized gels exhibited the same behavior as shown in Figures 2 and 4; although the neutral sample largely shrinks in the region where $20 \%<c<50 \%$, the mobility is largely suppressed when $c>50 \%$.
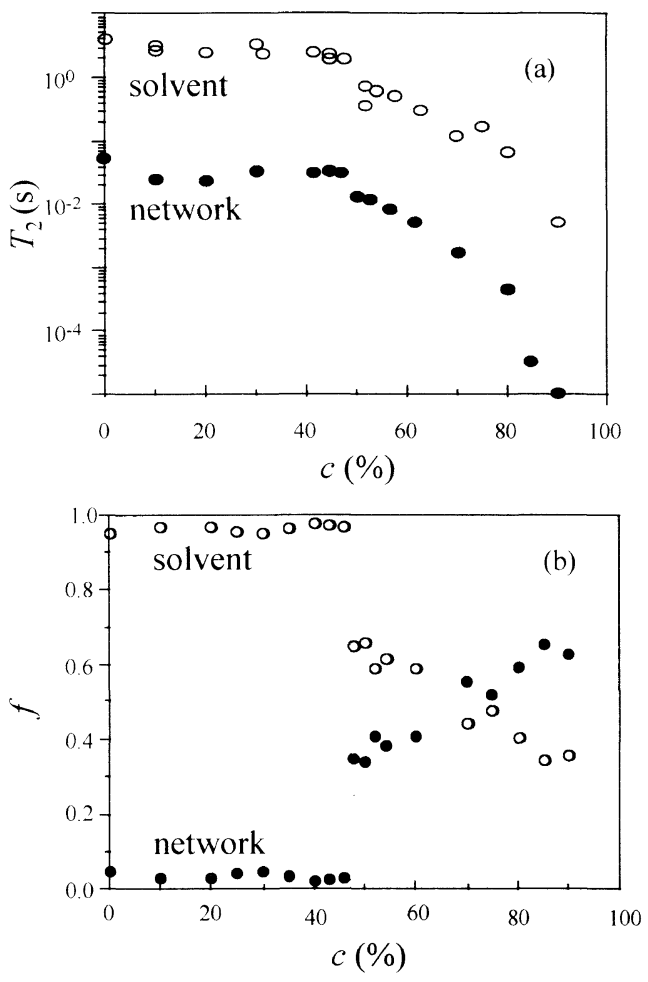

Figure 5. Dependence of $T_{2}$ (a) and fractional amount $f(\mathrm{~b})$ of each phase in the ionized gel on acetone content of the mixed solvent. The NMR signals were decomposed into two components.
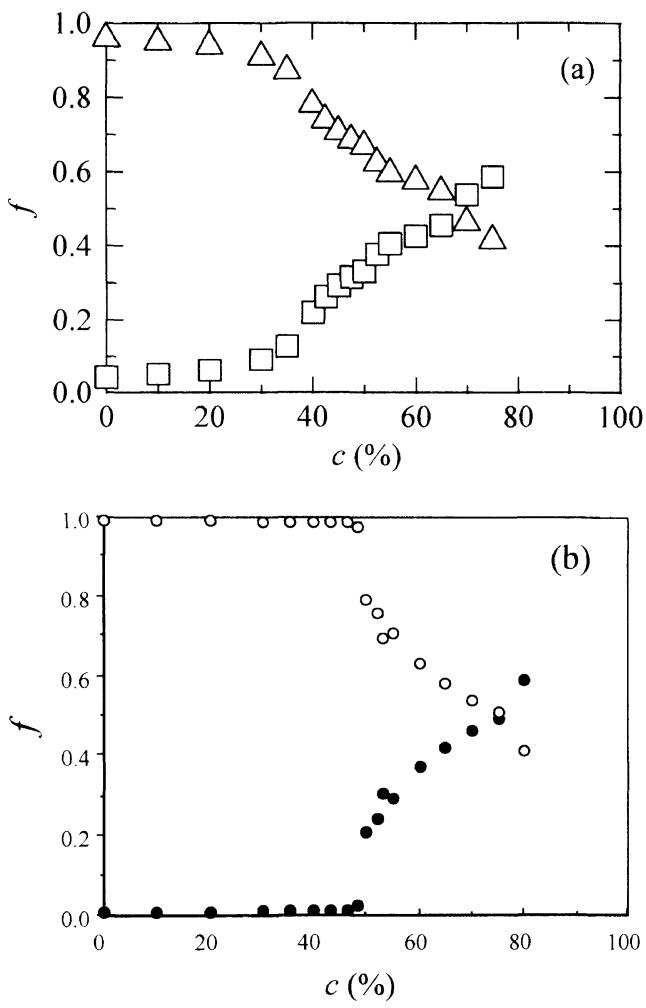

Figure 6. Proton molar fractions of the solvent and the network plotted against the acetone content of the mixed solvent for neutral (a) and ionized gels (b).

Figure 7 shows the time evolution of $V / V_{0}, T_{2}$, and $f$ in the shrinking process of an ionized gel. The sample that was first in the solvent with $c=45 \%$ was transferred into that with $c=52.5 \%$ (Figure 8 ). After the volume decreased a little in the early stage, it was almost constant 

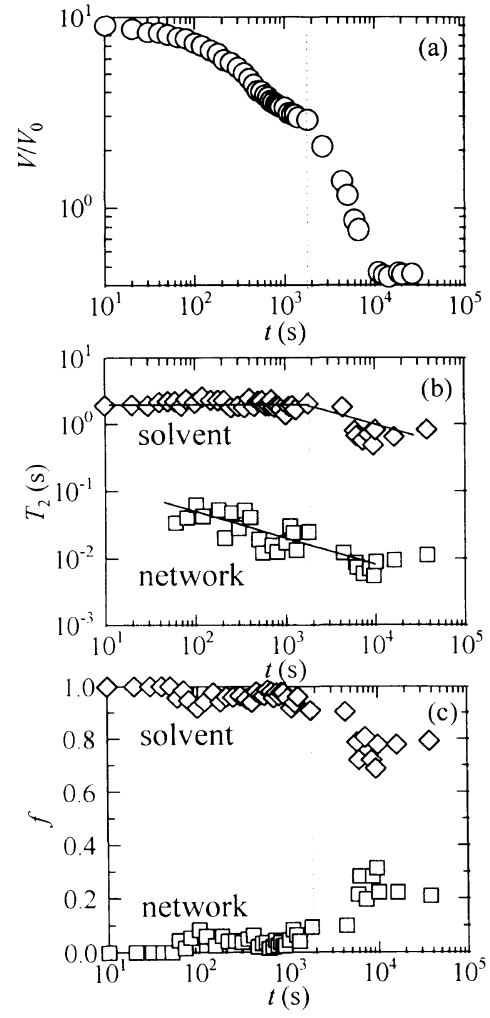

Figure 7. Time evolution of the swelling ratio (a), $T_{2}$ (b), and $f$ (c) for the shrinking process of acrylamide gels. The sample was transferred from a solvent with $c=45 \%$ into that with $52.5 \%$. The lines are the guides for the eye.

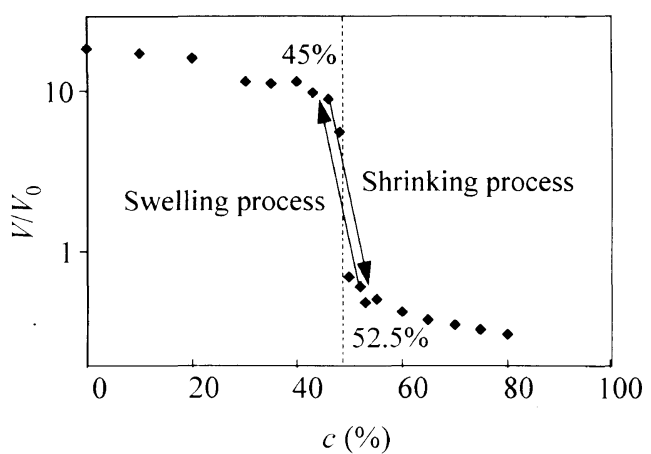

Figure 8. The conditions for the shrinking process and the swelling process of the ionized gel.

for a short period, and then it decreased again. The period with the almost constant swelling ratio is called the plateau period. ${ }^{26}$ This is attributed to the formation of a dense layer on the surface. After the relaxation of this structure during the plateau period, the volume further decreases. The value of $T_{2}$ of the solvent was almost constant in the early stage, then it decreased after the plateau period. The value of $T_{2}$ of the network polymer decreased constantly. The principal change in $f$ took place after the plateau period $\left(10^{3} \mathrm{~s}<t<10^{4} \mathrm{~s}\right)$.

Figure 9 shows the time evolution of $V / V_{0}, T_{2}$, and $f$ in the swelling process. The sample was transferred from the solvent with $c=52.5 \%$ into that with $c=45 \%$ (Figure 8 ). Contrary to the shrinking process, $T_{2}$ of the solvent increased constantly although that of the polymer was almost constant in the early stage. The time range where $T_{2}$ of the network polymer increased coincided with that where the volume largely increased $\left(10^{2} \mathrm{~s}<t<10^{3} \mathrm{~s}\right)$. The
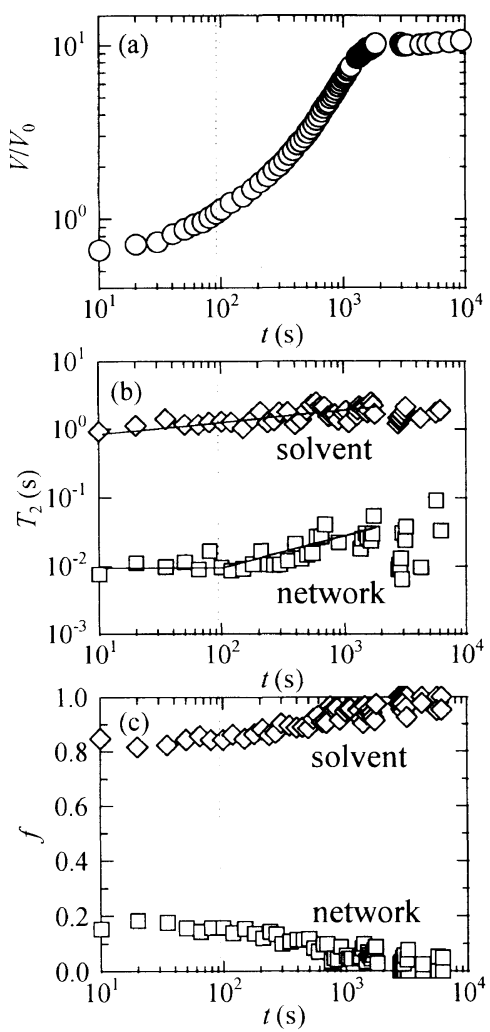

Figure 9. Time evolution of the swelling ratio (a), $T_{2}$ (b), and $f$ (c) for the swelling process of acrylamide gels. The sample was transferred from a solvent with $c=52.5 \%$ into that with $45 \%$. The lines are the guides for the eye.

principal change in $f$ took place during the major increase of the volume as well.

The environment of non-ionized gels whose diameter at the gelation was $1.55 \mathrm{~cm}$ was rapidly changed to form heterogeneous structures. The experimental conditions, the results of spin-spin relaxation measurement, and the sample codes are summarized in Table I. Since the inner and the outer parts of the heterogeneous samples were different in transparency and the texture, as schematically described in Figure 11, they were measured separately. The signals of $\mathrm{S}_{\mathrm{in}}, \mathrm{S} 0_{\text {out }}$, and $\mathrm{S} 35_{\text {out }}$ were decomposed into three components and the components with the shortest $T_{2}$ were Gaussian components, while the others were decomposed into two or three exponentially decaying components. Table II shows the proton molar fraction of the network polymer calculated from the mass of dried samples. Comparing the fractional amounts in Tables I and II, we concluded that the fastest and the second fastest decaying components are assigned to the network polymer for $\mathrm{S} 0_{\text {in }}$ and $\mathrm{S} 35_{\text {out }}$, and that the Gaussian component is assigned to the network for $\mathrm{S} 0_{\text {out }}$. Table III shows the results of $T_{1}$ measurement performed on the three samples with a Gaussian component in the transverse relaxation. The spin-lattice relaxation had two components. The difference in the number of components for $T_{1}$ and $T_{2}$ is ascribed to spin diffusion.

Figure 12(a) shows the recovery functions of magnetization in the hard phase obtained by the modified Goldman-Shen sequence. This experiment was performed on the three samples that had a fast decaying Gaussian component. The spin diffusion time $\tau$ of these curves was scaled by the characteristic time of diffusion 
Table I. Experimental conditions and the results of $T_{2}$ and the fractional amounts of heterogeneous samples. $c_{\text {init }} \rightarrow c_{\text {dest }}$ shows the initial and destination concentrations of the solvent. " $G$ " in the component 1 shows that it is a Gaussian component

\begin{tabular}{|c|c|c|c|c|c|c|}
\hline \multirow{2}{*}{ Condition } & & & \multicolumn{3}{|c|}{ Component } & \multirow{2}{*}{ Transparency } \\
\hline & & & 1 & 2 & 3 & \\
\hline \multirow{2}{*}{$0 \rightarrow 60 \%$} & $\mathrm{M} 0_{\text {in }}$ & $\begin{array}{l}T_{2}(\mathrm{~s}) \\
f(\%)\end{array}$ & $\begin{array}{c}1.5 \times 10^{-3} \\
25\end{array}$ & $\begin{array}{c}4.7 \times 10^{-2} \\
55\end{array}$ & $\begin{array}{c}3.2 \times 10^{0} \\
20\end{array}$ & Turbid \\
\hline & $\mathrm{M} 0_{\text {out }}$ & $\begin{array}{l}T_{2}(\mathrm{~s}) \\
f(\%)\end{array}$ & - & $\begin{array}{c}1.0 \times 10^{-3} \\
43\end{array}$ & $\begin{array}{c}2.0 \times 10^{-1} \\
57\end{array}$ & Transparent \\
\hline \multirow{2}{*}{$35 \rightarrow 60 \%$} & $\mathrm{M}_{35}$ in & $\begin{array}{l}T_{2}(\mathrm{~s}) \\
f(\%)\end{array}$ & $\begin{array}{c}2.7 \times 10^{-3} \\
23\end{array}$ & $\begin{array}{c}6.0 \times 10^{-2} \\
48\end{array}$ & $\begin{array}{c}3.5 \times 10^{0} \\
29\end{array}$ & Turbid \\
\hline & M35 & $\begin{array}{l}T_{2}(\mathrm{~s}) \\
f(\%)\end{array}$ & - & $\begin{array}{c}9.2 \times 10^{-4} \\
40\end{array}$ & $\begin{array}{c}1.6 \times 10^{-1} \\
60\end{array}$ & Transparent \\
\hline \multirow{2}{*}{$0 \rightarrow 100 \%$} & $\mathrm{~S} 0_{\text {in }}$ & $\begin{array}{l}T_{2}(\mathrm{~s}) \\
f(\%)\end{array}$ & $\begin{array}{c}7.1 \times 10^{-6} \\
\mathrm{G} 40\end{array}$ & $\begin{array}{c}3.2 \times 10^{-3} \\
26\end{array}$ & $\begin{array}{c}6.8 \times 10^{-1} \\
34\end{array}$ & Turbid \\
\hline & $\mathrm{S} 0_{\text {out }}$ & $\begin{array}{l}T_{2}(\mathrm{~s}) \\
f(\%)\end{array}$ & $\begin{array}{c}6.4 \times 10^{-6} \\
\text { G66 }\end{array}$ & $\begin{array}{c}1.8 \times 10^{-3} \\
30\end{array}$ & $\begin{array}{c}4.1 \times 10^{-1} \\
4\end{array}$ & Turbid \\
\hline \multirow{2}{*}{$35 \rightarrow 100 \%$} & $\mathrm{~S} 35_{\text {in }}$ & $\begin{array}{c}T_{2}(\mathrm{~s}) \\
f(\%)\end{array}$ & - & $\begin{array}{c}1.7 \times 10^{-2} \\
85\end{array}$ & $\begin{array}{c}7.4 \times 10^{0} \\
15\end{array}$ & Turbid \\
\hline & $\mathrm{S} 35_{\text {out }}$ & $\begin{array}{l}T_{2}(\mathrm{~s}) \\
f(\%)\end{array}$ & $\begin{array}{c}6.8 \times 10^{-6} \\
\text { G50 }\end{array}$ & $\begin{array}{c}1.9 \times 10^{-3} \\
20\end{array}$ & $\begin{array}{c}3.1 \times 10^{-1} \\
30\end{array}$ & Turbid \\
\hline
\end{tabular}

a not observed.

Table II. The proton molar fraction of the network $\left(f_{\mathrm{p}}\right)$ calculated from the mass of the dried samples

\begin{tabular}{ccccccccc}
\hline Sample & $\mathrm{M}_{\text {in }}$ & $\mathrm{M}_{\text {out }}$ & $\mathrm{M}^{2} 5_{\text {in }}$ & $\mathrm{M}^{2} 5_{\text {out }}$ & $\mathrm{S} 0_{\text {in }}$ & $\mathrm{S} 0_{\text {out }}$ & $\mathrm{S} 35_{\text {in }}$ & $\mathrm{S} 35_{\text {out }}$ \\
\hline$f_{\mathrm{p}} / \%$ & 27 & 61 & 32 & 55 & 77 & 75 & 25 & 61 \\
\hline
\end{tabular}

Table III. Results of $T_{1}$ and the fractional amounts of heterogeneous samples that had a Gaussian component in the transverse relaxation

\begin{tabular}{llrr}
\hline & & \multicolumn{2}{c}{ Component } \\
\cline { 3 - 4 } & & 1 & 2 \\
\hline $\mathrm{S} 0_{\text {in }}$ & $T_{1} / \mathrm{ms}$ & 104 & 1780 \\
& $f / \%$ & 60 & 40 \\
\hline $\mathrm{S} 0_{\text {out }}$ & $T_{1} / \mathrm{ms}$ & 89.7 & 1250 \\
& $f / \%$ & 91 & 9 \\
\hline & $T_{1} / \mathrm{ms}$ & 93.9 & 1660 \\
\hline & $f / \%$ & 67 & 33 \\
\hline
\end{tabular}

Table IV. Domain sizes of hard phases in heterogeneous gels

\begin{tabular}{lc}
\hline Sample & Domain size $/ \AA$ \\
\hline $\mathrm{S} 0_{\text {in }}$ & 120 \\
$\mathrm{~S} 0_{\text {out }}$ & 50 \\
$\mathrm{~S} 35_{\text {out }}$ & 60
\end{tabular}

$\tau^{* 1 / 2} \equiv \tau^{1 / 2} /\left[L /(2 d D)^{1 / 2}\right]$ as shown in Figure 12(b), where $L$ is the size of the hard phase, $d$ is the spatial dimension, and $D$ is the spin diffusion coefficient. The value of $D$ is obtained as $D=0.13 r_{0}^{2} / T_{2}$ where $r_{0}$ is the lattice constant. $^{27}$ With the scaling factor $s=\left(\tau^{*} / \tau\right)^{1 / 2}$, the domain size of the hard phase in the gel was calculated with a reference recovery curve of a block copolymer whose domain size is already known $(130 \AA){ }^{22}$ The results are summarized in Table IV. It should be noted that, since these samples are opaque, it would be difficult to apply light scattering to estimate the domain sizes.

\section{DISCUSSION}

Concentration Dependence of $T_{2}$

The transverse relaxation signals were decomposed into two components (the solvent and the network polymer) for both ionized and non-ionized samples for all $c$ (Figures 4 and 5). There must exist two states for the solvent molecules: those bound by the network and the free molecules. However, these two states were not detected by the pulsed NMR although the molecular mobility must be different from each other. This is ascribed to the fast exchange of solvent molecules between the two states. The observed spin-spin relaxation time $T_{2}^{(\text {obs })}$ is described as $T_{2}^{(\text {obs })^{-1}}=f^{(1)} / T_{2}^{(1)}+f^{(2)} / f_{2}^{(2)}$, where $f^{(i)}$ and $T_{2}^{(i)}$ are the fractional amount and $T_{2}$ in the state $i$, respectively. ${ }^{28}$ When gels are swollen and contain a lot of solvent inside, $T_{2}^{\text {(obs) }} \simeq T_{2}^{(\text {free solvent) }}$ since most of the solvent molecules are unbound.

As shown in Figures 2, 4, and 5, molecular mobility is more suppressed in the collapsed state $(c>50 \%$ for the non-ionized gel and $c>70 \%$ for the ionized gel) than in the concentration region where large collapse takes place $(30 \%<c<50 \%$ for the non-ionized gel and $c=49 \%$ for the ionized gel). There are two factors considered that suppress molecular mobility: volume of the system and the interaction parameter between the chains and the solvent. The results indicate that the interaction parameter has more influence on molecular 
mobility than the volume of the system. As the mobility of the network polymer is suppressed, that of the solvent trapped in the mesh is also suppressed. Another possible reason is that. in the present experiment, the solvent is a mixture of acetone and water. A sufficient amount of water persists in the solvent around the volume phase transition point. These water molecules interact with the network chains. There is good possibility that they increase the mobility. Molecular mobility detected by NMR reflects the local environment. One cannot discuss the mobility only with the volume itself. In the present case, further discussions should be based on the ternary system of the polymer, water, and acetone.

There are a few works that showed the network polymers have sufficient mobility in the collapsed state near the transition point. ${ }^{7.11}$ Tokuhiro et al. compared ionized and non-ionized $N$-isopropylacrylamide gels and concluded that the ionization increases molecular mobility in the contracted gels. In the present study, however, the network chains of both ionized and non-ionized samples are mobile in the collapsed state. This indicates that the influence of ionization on the enhancement of mobility is small in our case.

\section{Shrinking and Swelling Dynamics}

Dynamics of the shrinking and swelling process is different from each other as shown in Figures 7 and 9. In the shrinking process, the response of the network chains is faster than that of the solvent. In the swelling process, on the other hand, the response of the solvent is faster than that of the polymer. This reflects the behavior that the swelling and shrinking start on the boundary of the samples as discussed below.

The shrinking process is divided into two stages: before the end of the plateau period (stage I) and after the end of this period (stage II). In stage I, where only the outer part of the gel shrinks (Figure 10(a)), $T_{2}$ of the solvent is almost constant and that of the polymer decreases. There are several factors to explain this behavior. The reason for the fast response of the network is that, when a gel is transferred from a good solvent to a poor one, the polymer chains contact to each other to reduce contact free energy and, hence, the mobility of the polymer is lowered. The density of the network chains is larger in the outer part than in the inner part. The difference in mobility of the chains between the inner and outer parts is not large enough for pulsed NMR to decompose. The decrease in $T_{2}$ for the chains reflects the superposition of the increasing amount of immobile chains (outer part) and the decreasing amount of mobile ones (inner part). The reason for the almost constant $T_{2}$ of the solvent in stage $I$ is the presence of a lot of free (unbound) solvent molecules in the swollen inner part. Pulsed NMR again cannot decompose the signals of the solvent in the collapsed and still swollen networks. In stage II, where the volume further decreased, the decrease in $T_{2}$ of the solvent reflects the increasing amount of the bound molecules and decreasing amount of the free molecules in the shrinking process.

The swelling process is also divided into two stages: before (stage $\mathrm{I}^{\prime}$ ) and after (stage $\mathrm{II}^{\prime}$ ) the beginning of the increase in $T_{2}$ of the network. In stage $I^{\prime}$, where the total volume changes little, the outer part swells first, while (a)

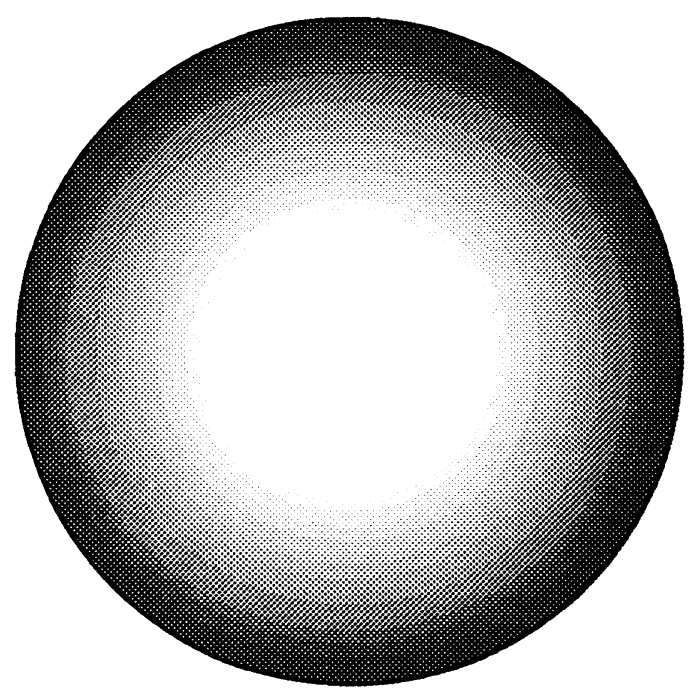

(b)

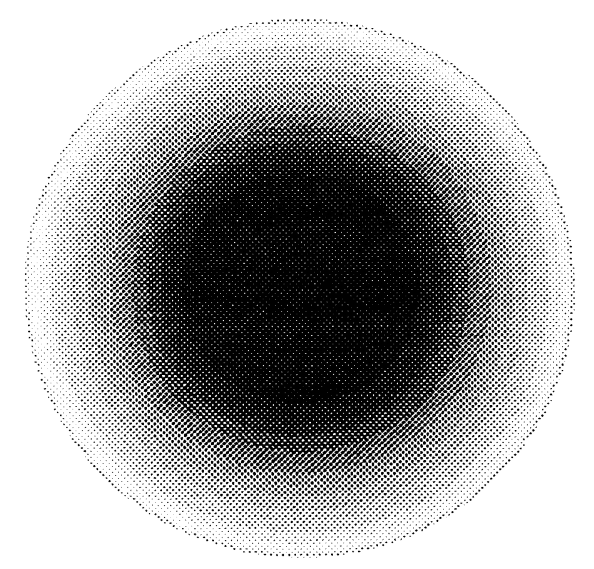

Figure 10. Schematic cross sections of the gels in the shrinking process (a) and the swelling process (b). The gray scale shows the density of the network; the shaded part is collapsed and the white part is swollen.

the inner part is still collapsed (Figure 10(b)). Since the amount of the polymer in the outer swollen part is small, the total $T_{2 \mathrm{~S}}$ changes little. On the other hand, this part contains a sufficient amount of the solvent, the amount of which is comparable to that in the inner part. The solvent in the swollen outer part increases the mobility of the solvent in stage $\mathrm{I}^{\prime}$. In stage $\mathrm{II}^{\prime}$, where the total volume largely increases, $T_{2}$ of the network begins to increase. As the volume increases, the amount of mobile chains in swollen networks increases.

\section{Heterogeneous Structure}

Transparency and the texture of the inner and the outer parts of the heterogeneous samples were different as shown in Figure 11. The difference is ascribed to the shrinking behavior of gels that the outer part shrinks first. This is a consequence of the cooperative diffusive motion of gels. ${ }^{13.14}$ It is easier for the outer part than the inner part to approach the equilibrium collapsed state for the kinetic reason. The transparency of the samples $\mathrm{M} 0_{\text {out }}$ and $\mathrm{M} 35_{\text {out }}$ shows that they have reached the equilibrium state. This is reflected by the results of the transverse relaxation experiment on these samples (Table I). They are almost the same as those in Figure 4. On the other hand, both the outer and the inner parts were 
(a)

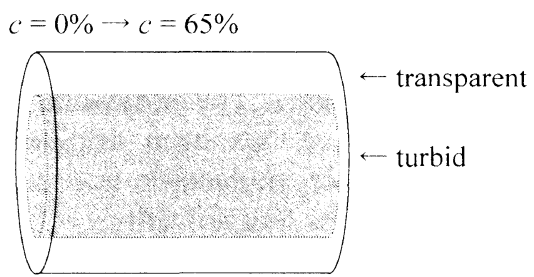

(b)

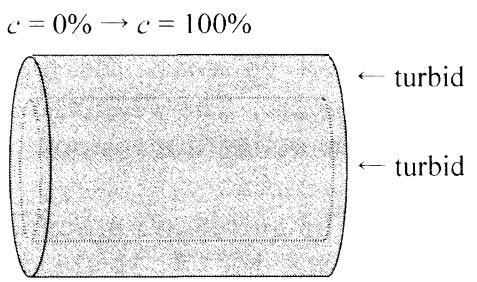

Figure 11. Schematic figure of the difference of the outer and inner parts of a cylindrical gel after an abrupt change of the environment for samples M0 (a) and S0 (b)

opaque for samples S0 and S35. However, the textures were different; the inner parts were softer and coarser than the outer parts. The reason for the difference is described as follows: the abrupt change of the environment makes the contracted outer part hard enough to prevent further collapse. The inner part still contains a sufficient amount of the poor solvent to which the hard outer part is impermeable. The excess amount of the poor solvent in the inner part forces more network chains to contact to each other to reduce the contact free energy between the polymer and the solvent. This is reflected by the difference in the domain sizes of $\mathrm{S} 0_{\text {in }}$ and $\mathrm{S} 0_{\text {out }}$ (Table IV).

The fast collapse of the outer part also explains that S35 in has only two components in the transverse relaxation. Since the volume of the non-ionized sample in the solvent with $c=35 \%$ is smaller than in the pure water by $40 \%$ (Figure 2), its mesh size is also smaller. When it is soaked into pure acetone, the immediate collapse of the outer part hinders the exchange of the solvent in the inner part. The two components of S35 in are the consequence of the less poor solvent in the inner part.

The transverse relaxation curves of $\mathrm{S}_{i}, \mathrm{~S}_{\text {out }}$, and S35 out were decomposed into three components (Table I). The component whose $T_{2}$ is of the order of $10^{-5} \mathrm{~s}$ is assigned to a solid-like state in the heterogeneous structure. On the other hand, the solvent molecules, which have long $T_{2}$, are divided into two types: one is those in the dense region of the network and the other is those in the sparse region (Figure 13). There is little possibility that these regions frequently exchange the solvent molecules and that it has sufficient influence on the transverse relaxation behavior. The most mobile component, therefore, is assigned to the solvent in the sparse regions and a part of the intermediate component is assigned to that in the dense regions. In both regions, there are unbound and bound solvent. A large fraction of the solvent in the sparse regions is unbound and only a small fraction is bound by the network. The situation is the opposite in the dense regions.

Gels with heterogeneous structure are white and turbid. The reason for the turbidity is that light is
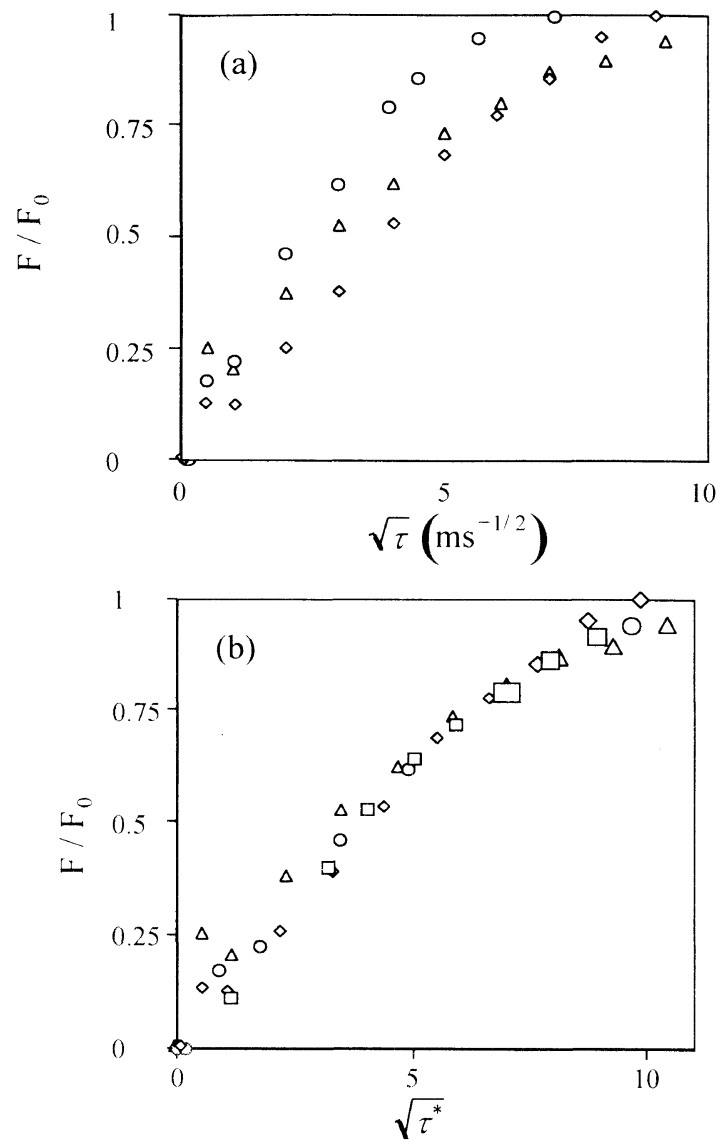

Figure 12. Recovery curves obtained by Goldman-Shen sequence from the samples (a) and those scaled to that of a block copolymer (b) for the samples $S 0_{\text {in }}(\diamond), S 0_{\text {out }}(\bigcirc)$, and $\mathrm{S} 35(\triangle)$. The symbol $\square$ in (b) is a recovery function of a block copolymer whose domain size is already known. 22

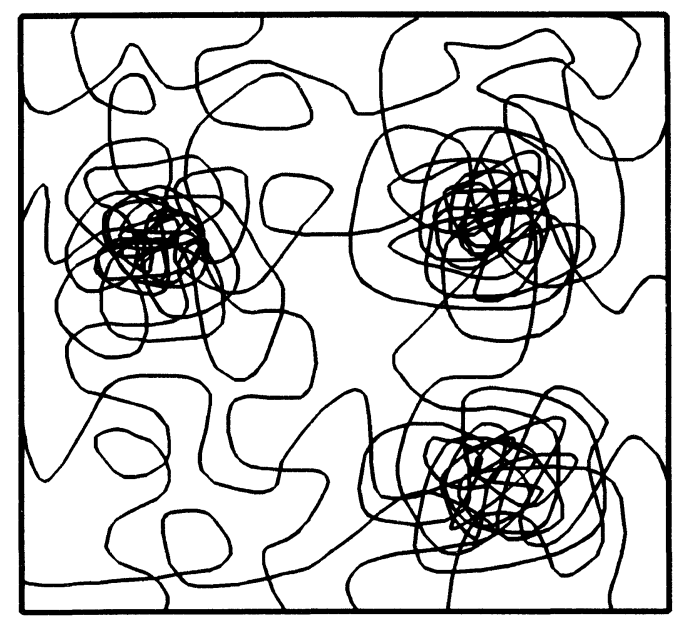

Figure 13. A schematic figure of the network structure of a heterogeneous gel.

scattered by the heterogeneity in the refractive index in the sample. This indicates that the dimension of the heterogeneous structure is comparable to the wave length of visible light. The results of the size of the hard phase obtained by the Goldman-Shen experiment is smaller than is estimated from this consideration. This is because the hard phase detected by pulsed NMR is on a smaller scale. The heterogeneous structure detected by light scattering is heterogeneity of the gel itself (network chains and the solvent). However, the hard phase detected by 
the solid echo pulse sequence is a smaller solid-like structure whose $T_{2}$ is of the order of $10^{-5}$. Solid-like regions that are aggregations of only the chains appear in the dense phase in Figure 13.

We now discuss the influence of the interface between the hard solid-like regions and the surrounding soft regions. Figure 14 shows two types of interfaces: one is that with a gradual change of the structure in a real system (Figure 14(a)) and the other is an ideal stepwise structure (Figure 14(b)). After the magnetization in the hard phase is eliminated by the Goldman-Shen sequence, the interface still has appreciable magnetization since $T_{2}$ in this phase is longer than that in the hard phase. Only the boundaries of the hard regions have magnetization for a short diffusion time $\tau$. Since they are less perfect than the hard phase, the value of $T_{2}$ for the hard phase is longer for small $\tau$ than that for large $\tau$ as shown in
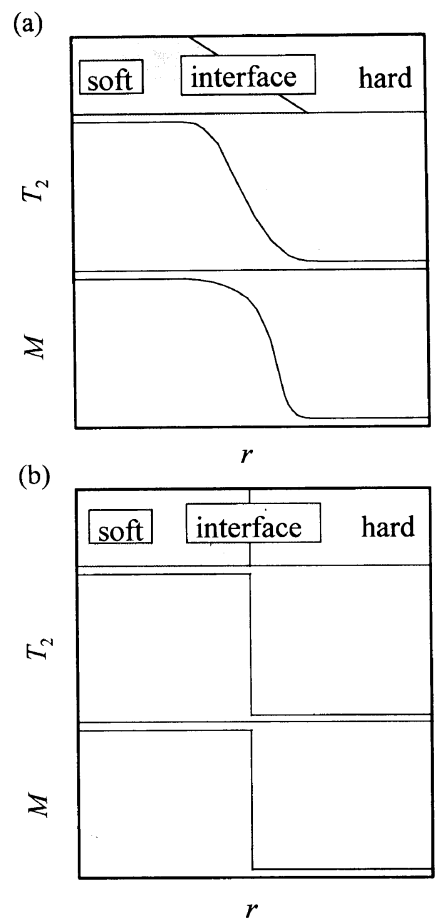

Figure 14. Spatial profiles of $T_{2}$ and the magnetization after the second pulse $\left(90_{-x}^{\circ}\right)$ in the Goldman-Shen sequence for a real system (a) and an ideal system (b).

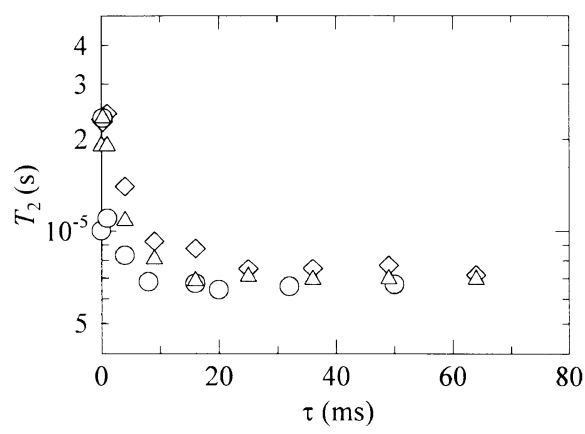

Figure 15. Dependence of $T_{2}$ of the hard phases on the spin diffusion time $\tau$. Symbols are identical with those in Figure 12 .
Figure 15. Moreover, the interfacial phase contains a small amount of solvent molecules. The origin of spin diffusion is spin flip-flop motion through the dipoledipole interaction of nuclei. The polymeric systems the Goldman-Shen sequence has been applied to so far contained only polymer molecules and no low molecular-weight substances like solvent. ${ }^{20-22}$ In such situations, one has to consider intra- and intermolecular spin diffusion in polymers. In the present study, however, the self-diffusion of the solvent molecules must be taken into account. This effect enhances the magnetization recovery in the hard phase. The obtained dimensions may be smaller than the actual dimensions.

Acknowledgment. The autors are grateful to Mr. Y. Nakanishi, Mr. T. Mitsui, Mr. A. Hotta, and Mr. H. Yamaguchi for their valuable assistance.

\section{REFERENCES}

1. T. Tanaka, Phys. Rev. Lett., 40, 820 (1978).

2. T. Tanaka, D. J. Fillmore, S.-T. Sun, and I. Nishio, Phys. Rev. Lett., 45, 1636 (1980).

3. S. Hirotsu, Y. Hirokawa, and T. Tanaka, J. Chem. Phys., 87, 1392 (1987).

4. Y. Hirokawa, T. Tanaka, and S. Katayama, in "Microbial Adhesion and Aggregation," K. C. Marshall Ed., Springer-Verlag, Berlin/Heidelberg, 1984.

5. I. Ohmie and T. Tanaka, J. Chem. Phys., 77, 5725 (1982).

6. S. Katayama, S. Kazama, and H. Yoshioka, J. Phys. Chem., 96, 2023 (1992)

7. Y. Hu, K. Horie, H. Ushiki, F. Tsunomori, and T. Yamashita, Macromolecules, 25, 7324 (1992).

8. Y. Hu, K. Horie, and H. Ushiki, Macromolecules, 25, 6040 (1992).

9. Y. Hu, K. Horie, H. Ushiki, T. Yamashita, and F. Tsunomori, Macromolecules, 26, 1761 (1993).

10. M. Corti, L. Pavesi, A. Rigamonti, and F. Tabak, Phys. Rev. A, 43, 6887 (1991).

11. T. Tokuhiro, T. Amiya, A. Mamada, and T. Tanaka, Macromolecules, 24, 2936 (1991).

12. T. Hayashi, Y. Urai, J. Inoue, T. Ikehara, and T. Nishi, Rep Prog. Polym. Phys. Jpn., 35, 499 (1992).

13. T. Tanaka and D. J. Fillmore, J. Chem. Phys., 70, 1214 (1979).

14. Y. Li and T. Tanaka, J. Chem. Phys., 92, 1365 (1990).

15. Y. Li, G. Wang, and Z. Hu, Macromolecules, 28, 4194 (1995).

16. H. Tanaka, K. Fukumori, and T. Nishi, J. Chem. Phys., 89, 3363 (1988).

17. T. Shiga, K. Fukumori, Y. Hirose, A. Okada, and T. Kurauchi, J. Polym. Sci., B, Polym. Phys., 32, 85 (1994).

18. F. Tabak, M. Corti, L. Pavesi, and A. Rigamonti, J. Phys. C, 20, 5691 (1987)

19 D. C. Douglass and V. J. McBrierty, J. Chem. Phys., 54, 4085 (1971).

20. R. A. Assink, Macromolecules, 11, 1233 (1978).

21. T. T. P. Cheung, J. Chem. Phys., 76, 1248 (1982).

22. H. Tanaka and T. Nishi, Phys. Rev. B, 33, 32 (1986).

23. M. Goldman and L. Shen, Phys. Rev., 144, 321 (1966).

24. S. Meiboom and D. Gill, Rev. Sci. Instrum., 29, 688 (1958).

25. J. G. Powles and J. H. Strange, Proc. Phys. Soc. London, 82, 6 (1963).

26. E. S. Matsuo and T. Tanaka, J. Chem. Phys., 89, 1695 (1988).

27. T. T. P. Cheung, B. C. Gerstein, L. M. Ryan, R. E. Taylor, and C. R. Dybowski, J. Chem. Phys., 73, 6059 (1980).

28. J. R. Zimmermann and W. E. Brittin, J. Phys. Chem., 61, 1327 (1957) 